Authorship note: RTC and SKC contributed equally to this work.

Conflict of interest: LTF has served as a consultant for Theratechnologies, Inc. JMB reports research funding from Boehringer Ingelheim. SHJS has received funding from AstraZeneca and Boehringer Ingelheim to support bioinformatics analysis and software development through her service as the director of the Harvard Chan Bioinformatics Core. KEC reports research funding from Boehringer Ingelheim and has served as a consultant to Novo Nordisk, Inc. and Bristol Myers Squibb (BMS). TLS reports research funding from Novo Nordisk, Inc. RTC reports research funding from AbbVie, Gilead, Merck, BMS, Boehringer Ingelheim, Janssen, and Roche. SKC has served as a consultant (personal) and currently serves as a consultant through an Institutional Consulting Agreement with Theratechnologies. The Massachusetts General Hospital has a royalty and license agreement with Theratechnologies for tesamorelin. SKG is the inventor on a pending patent, GHRH or Analogues Thereof For Use in Treatment of Hepatic Disease (application 16832128).

Submitted: May 11, 2020

Accepted: July 15, 2020

Published: August 20, 2020.

Reference information: JCI Insight. 2020;5(16):e140134 https://doi.org/10.1172/jci. insight.140134.

Copyright: (c) 2020, Fourman et al. This is an open access article published under the terms of the Creative Commons Attribution 4.0 International License.

\section{Effects of tesamorelin on hepatic transcriptomic signatures in HIV-associated NAFLD}

Lindsay T. Fourman, ${ }^{1}$ James M. Billingsley, ${ }^{2}$ George Agyapong, ${ }^{3}$ Shannan J. Ho Sui, ${ }^{2}$ Meghan N. Feldpausch, ${ }^{1}$ Julia Purdy, ${ }^{4}$ Isabel Zheng, ${ }^{1}$ Chelsea S. Pan, ${ }^{1}$ Kathleen E. Corey, ${ }^{3}$ Martin Torriani, ${ }^{5}$ David E. Kleiner, ${ }^{6}$ Colleen M. Hadigan, ${ }^{4}$ Takara L. Stanley, ${ }^{1}$ Raymond T. Chung, ${ }^{3}$ and Steven K. Grinspoon ${ }^{1}$

${ }^{1}$ Metabolism Unit, Massachusetts General Hospital and Harvard Medical School, Boston, Massachusetts, USA. ${ }^{2}$ Harvard Chan Bioinformatics Core, Department of Biostatistics, Harvard T.H. Chan School of Public Health, Boston, Massachusetts, USA. 'iver Center, Digestive Healthcare Center, Massachusetts General Hospital and Harvard Medical School, Boston, Massachusetts, USA. ${ }^{4}$ National Institute of Allergy and Infectious Diseases, NIH, Bethesda, Maryland, USA. ${ }^{5}$ Department of Radiology, Massachusetts General Hospital and Harvard Medical School, Boston, Massachusetts, USA. ${ }^{6}$ Laboratory of Pathology, Center for Cancer Research, National Cancer Institute, NIH, Bethesda, Maryland, USA.

Nonalcoholic fatty liver disease (NAFLD) is a common comorbidity among people living with HIV that has a more aggressive course than NAFLD among the general population. In a recent randomized placebo-controlled trial, we demonstrated that the growth hormone-releasing hormone analog tesamorelin reduced liver fat and prevented fibrosis progression in HIVassociated NAFLD over 1 year. As such, tesamorelin is the first strategy that has shown to be effective against NAFLD among the population with HIV. The current study leveraged paired liver biopsy specimens from this trial to identify hepatic gene pathways that are differentially modulated by tesamorelin versus placebo. Using gene set enrichment analysis, we found that tesamorelin increased hepatic expression of hallmark gene sets involved in oxidative phosphorylation and decreased hepatic expression of gene sets contributing to inflammation, tissue repair, and cell division. Tesamorelin also reciprocally up- and downregulated curated gene sets associated with favorable and poor hepatocellular carcinoma prognosis, respectively. Notably, among tesamorelin-treated participants, these changes in hepatic expression correlated with improved fibrosis-related gene score. Our findings inform our knowledge of the biology of pulsatile growth hormone action and provide a mechanistic basis for the observed clinical effects of tesamorelin on the liver.

\section{Introduction}

Nonalcoholic fatty liver disease (NAFLD) has become a leading cause of liver disease worldwide. NAFLD encompasses a broad spectrum of disease that ranges histologically from simple steatosis to steatohepatitis (NASH) to fibrosis and may ultimately progress to cirrhosis or hepatocellular carcinoma (HCC). NAFLD is a major comorbidity among people living with HIV (PLWH), with over one-third of individuals affected (1). Compared with the general population, PLWH have a more aggressive disease course that is characterized by a greater prevalence of NASH and fibrosis as well as an accelerated rate of fibrosis progression (2).

Despite the heightened burden of NAFLD in HIV, approved pharmacologic interventions to treat this condition in HIV are lacking. Tesamorelin, a hypothalamic growth hormone-releasing hormone (GHRH) analog that augments endogenous pulsatile growth hormone $(\mathrm{GH})$ and downstream insulin-like growth factor-1 (IGF-1) secretion, is FDA-approved to reduce visceral adiposity in HIV. In a recent randomized placebo-controlled trial, we investigated for the first time to our knowledge the effects of this agent on liver fat and histology among individuals with HIV-associated NAFLD. Through this work, we found that tesamorelin significantly reduced liver fat and prevented fibrosis progression over 1 year (3).

While high-throughput gene expression technologies have been widely used to elucidate key pathways predictive of NAFLD course among the general population, few studies have examined the effects 
of potential therapeutic strategies on hepatic transcriptomic signatures in any patient group. In the current study, we leveraged paired liver biopsy specimens from our recent clinical trial to investigate differential changes in hepatic gene expression by tesamorelin versus placebo over 1 year. Through this analysis, we identified novel biological pathways changing in tesamorelin versus placebo that may underlie the phenotypic changes that we observed in our clinical trial. Our findings expand upon the known effects of tesamorelin on hepatic pathophysiology, using a transcriptomic approach to identify effects on gene signatures encompassing major inflammatory and fibrotic pathways and increasing our understanding of the physiologic effects of augmented pulsatile GH secretion on the liver transcriptome.

\section{Results}

\section{Characteristics of study participants}

Demographic and clinical characteristics were similar between treatment groups. Data for individual treatment groups are shown in Table 1 . In the overall sample, participants (53 \pm 7 years old, $77 \%$ male) had chronic HIV infection (17 \pm 9 years) with excellent virologic control. All subjects were on stable antiretroviral therapy (ART), with 64\% receiving integrase inhibitor-based regimens. Baseline hepatic fat fraction in our cohort was $14 \% \pm 9 \%$. Moreover, $31 \%$ and $41 \%$ of participants had histologically defined NASH and fibrosis, respectively. Over the study period, changes in body weight, dietary intake, and other relevant clinical factors were not found to differ between treatment groups, as has been previously described (3). Baseline characteristics of the participants included in this analysis with transcriptomic data on paired biopsy specimens did not differ substantially from that of the participants in the larger parent trial (see Supplemental Table 1; supplemental material available online with this article; https://doi.org/10.1172/jci.insight.140134DS1).

\section{Effects of tesamorelin on expression of hallmark gene sets}

We first performed an unbiased analysis to identify hepatic biological pathways differentially modulated by tesamorelin versus placebo using gene set enrichment analysis (GSEA), which determines whether a priori-defined sets of genes defining distinct biological pathways are differentially expressed between 2 conditions. In pathways that are significantly enriched at either the top or bottom of a transcriptome ranked by differential expression, the leading edge genes are the subset of genes that contribute most to the enrichment signal. For target gene sets, we queried the Molecular Signatures Database (MSigDB) hallmark gene sets (4), which consist of 50 gene sets that represent well-defined biological states or processes (5). Using this approach, we found 14 hallmark gene sets to be differentially regulated between treatment groups with false discovery rate (FDR) $q$ value less than 0.05 (Table 2 and Figure 1). In this regard, a gene set encoding oxidative phosphorylation proteins was upregulated by tesamorelin versus placebo. Moreover, 13 gene sets pertaining to inflammation, tissue repair, and cell division were downregulated in the tesamorelin group relative to the placebo group. There was minimal overlap of leading edge genes between the differentially modulated gene sets (Supplemental Figure 1).

Cell metabolism. The OXIDATIVE_PHOSPHORYLATION hallmark gene set was differentially regulated between tesamorelin and placebo (normalized enrichment score $[\mathrm{NES}]=1.94$, FDR $q$ value $=0.0005$ ). In particular, we found that genes involved in oxidative phosphorylation were globally upregulated among tesamorelin-treated participants and downregulated among placebo-treated participants (Figure 1 and Figure 2). A large proportion of leading edge genes within this gene set encoded subunits of the electron transport chain (Figure 3). Examples included NDUFA6 and NDUFB1 of complex I, SDHC and SDHD of complex II, UQCR 10 and UQCRH of complex III, COX7A2L and COX17 of complex IV, and ATP5PF and ATP5F1C of complex V. Relatedly, other genes within the leading edge supported the electron transport chain by participating in the import and insertion of transmembrane proteins into the mitochondrial inner membrane, such as TIMM8B and TIMM9. Last, a subset of leading edge genes encoded enzymes involved in cell catabolism, including $E C I 1$ and $E C H S 1$ of the fatty acid $\beta$-oxidation pathway and $F H$ and $I D H 3 B$ of the tricarboxylic acid cycle. Among all participants, changes in expression of oxidative phosphorylation genes related to changes in IGF-1 transcription ( $r=0.35, P=0.03)$, change in serum IGF-1 $(r=0.45, P=0.005)$, and change in visceral fat content $(r=-0.42, P=0.008)$ but not to change in fasting glucose $(r=0.06, P=0.7)$.

Inflammation. Five hallmark gene sets pertaining to inflammation were differentially regulated by tesamorelin versus placebo: TNFA_SIGNALING_VIA_NFKB $($ NES $=-1.78$, FDR $q$ value $=0.01)$, IL6_JAK_STAT3_SIGNALING $($ NES $=-1.71$, FDR $q$ value $=0.02)$, ALLOGRAFT_REJECTION 
Table 1. Baseline demographic and clinical characteristics

\begin{tabular}{|c|c|c|}
\hline & Tesamorelin $(n=18)$ & Placebo $(n=21)$ \\
\hline Age (years) & $53 \pm 7$ & $53 \pm 8$ \\
\hline$\%$ male/female & $72 / 28$ & $81 / 19$ \\
\hline \multicolumn{3}{|l|}{$\%$ race } \\
\hline White & 56 & 62 \\
\hline Black & 39 & 33 \\
\hline Other & 6 & 5 \\
\hline \% Hispanic & 11 & 5 \\
\hline Duration of HIV infection (years) & $15 \pm 9$ & $18 \pm 9$ \\
\hline CD4 count (cells/mm³) & $715 \pm 262$ & $795 \pm 273$ \\
\hline Log HIV viral load & $0.29 \pm 0.56$ & $0.50 \pm 0.75$ \\
\hline \multicolumn{3}{|l|}{$\%$ current antiretroviral use } \\
\hline NRTI & 83 & 100 \\
\hline $\mathrm{Pl}$ & 28 & 24 \\
\hline NNRTI & 39 & 33 \\
\hline Integrase inhibitor & 72 & 57 \\
\hline \% type 2 diabetes & 6 & 10 \\
\hline Hepatic fat fraction (\%) & $13 \pm 8$ & $15 \pm 10$ \\
\hline$\%$ NASH & 28 & 33 \\
\hline$\%$ fibrosis & 44 & 38 \\
\hline Stage 1 & 17 & 14 \\
\hline Stage 2 & 17 & 14 \\
\hline Stage 3 & 11 & 10 \\
\hline Body mass index $\left(\mathrm{kg} / \mathrm{m}^{2}\right)$ & $30.6 \pm 7.0$ & $33.0 \pm 5.4$ \\
\hline Waist circumference (cm) & $109 \pm 16$ & $114 \pm 11$ \\
\hline Visceral adipose tissue area $\left(\mathrm{cm}^{2}\right)$ & $235 \pm 100$ & $256 \pm 112$ \\
\hline $\begin{array}{l}\text { Daily caloric intake per kilogram body } \\
\text { weight (kcal/kg) }\end{array}$ & $20 \pm 8$ & $24 \pm 7$ \\
\hline \multicolumn{3}{|c|}{$\begin{array}{l}\text { There were no statistically significant differences between groups at baseline for any of the variables shown above. } \\
\text { Continuous variables are presented as mean } \pm \text { standard deviation. NASH, nonalcoholic steatohepatitis; NNRTI, non- } \\
\text { nucleoside reverse transcriptase inhibitor; NRTI, nucleoside reverse transcriptase inhibitor; PI, protease inhibitor. }\end{array}$} \\
\hline
\end{tabular}

$(\mathrm{NES}=-1.62$, FDR $q$ value $=0.03)$, INFLAMMATORY_RESPONSE $(\mathrm{NES}=-1.50$, FDR $q$ value $=$ $0.03)$, and IL2_STAT5_SIGNALING (NES $=-1.48$, FDR $q$ value $=0.03)$. Overall, all 5 gene sets were downregulated in the tesamorelin group and upregulated in the placebo group (Figure 4). Collectively, the leading edges of these gene sets comprised genes involved in the function of the innate and adaptive immune response. These included genes encoding immune cell components, such as $C D 8 B, C D 3 D$, and $C D 4$, which are involved in $\mathrm{T}$ cell receptor activation, and $H L A-D Q A 1$, which is involved in antigen presentation. In addition, genes needed for recruitment and maintenance of immune cells at sites of tissue injury were also prominent within the leading edges of the inflammatory gene sets. Examples were CCL5 and CCL20 encoding cytokines chemotactic for diverse immune cell types, ICAM1 encoding a cell surface adhesion molecule important for leukocyte transendothelial migration, and IL15RA mediating survival of $\mathrm{T}$ cells and natural killer cells.

Tissue repair. Four hallmark gene sets related to tissue repair were differentially regulated by tesamorelin versus placebo: TGF_BETA_SIGNALING (NES $=-1.67$, FDR $q$ value $=0.03)$, APOPTOSIS $(\mathrm{NES}=-1.56$, FDR $q$ value $=0.03)$, UV_RESPONSE_DN $(\mathrm{NES}=-1.50$, FDR $q$ value $=0.03)$, and EPITHELIAL_MESENCHYMAL_TRANSITION (NES $=-1.46$, FDR $q$ value $=0.04)$. In general, all 4 gene sets were downregulated among tesamorelin-treated participants, whereas they were upregulated among the placebo-treated arm (Figure 4). Within the leading edges of these gene sets were genes important for extracellular matrix (ECM) remodeling, including BGN, SERPINH1, and COL1A1 responsible for collagen biosynthesis and assembly, MMP14 and MMP2 encoding metalloproteinases that mediate ECM degradation, and TIMP1 encoding a metalloproteinase inhibitor. Also included within the leading edges of these gene sets were genes that contribute to epithelial-mesenchymal transition (EMT), such as $D A B 2$, 
Table 2. Hallmark gene sets modulated by tesamorelin versus placebo

\begin{tabular}{|c|c|c|c|}
\hline Gene set name & Gene set description & FDR q value & Top leading edge genes \\
\hline \multicolumn{4}{|c|}{ Upregulated by tesamorelin relative to placebo } \\
\hline \multicolumn{4}{|l|}{ Cell metabolism } \\
\hline OXIDATIVE_PHOSPHORYLATION & $\begin{array}{l}\text { Genes encoding proteins } \\
\text { involved in oxidative } \\
\text { phosphorylation }\end{array}$ & 0.0005 & $\begin{array}{l}\text { TIMM8B, ATP5PF, TIMM17A, OAT, COX7A2L, NDUFA6, } \\
\text { NDUFB1, VDAC2, SURF1, ATP5F1C, MCST3, MTX2, UQCR10, } \\
\text { UQCRH, CASP7, NDUFB6 NDUFS4, ATP6V0B, COX17, UQCRQ }\end{array}$ \\
\hline \multicolumn{4}{|c|}{ Downregulated by tesamorelin relative to placebo } \\
\hline IL6_AK_STAT3_SIGNALING & $\begin{array}{l}\text { Genes upregulated by IL- } 6 \text { via } \\
\text { STAT3, e.g., during acute phase } \\
\text { response }\end{array}$ & 0.02 & $\begin{array}{c}\text { IL15RA, TNFRSF21, CXCL3, ITCB3, IFNGR2, CSF1, CD9, IL9R, } \\
\text { PIM1, LEPR, IL1R2, PIK3R5, IL4R, CNTFR, JUN, CSF3R, } \\
\text { TGFB1, EBI3, TNFRSF1A, IL17RB }\end{array}$ \\
\hline ALLOGRAFT_REJECTION & $\begin{array}{l}\text { Genes upregulated during } \\
\text { transplant rejection }\end{array}$ & 0.03 & $\begin{array}{c}\text { ZAP70, TAP2, CD7, CD8B, NCR1, CD3D, CCL5, ABCE1, ELF4, } \\
\text { ICAM1, CDKN2A, TLR3, TLR6, CCL22, CRTAM, HLA-DQA1, } \\
\text { ICSF6, GPR65, WARS, LY86 }\end{array}$ \\
\hline IL2_STAT5_SIGNALING & $\begin{array}{l}\text { Genes upregulated by STAT5 in } \\
\text { response to IL-2 stimulation }\end{array}$ & 0.03 & $\begin{array}{l}\text { TWSG1, ARL4A, ALCAM, IKZF4, S100A1, HK2, BHLHE40, } \\
\text { GPR65, IFITM3, AHCY, SCN9A, SMPDL3A, SLC1A5, RCS16, } \\
\text { BATF3, FAM126B, SELL, DCPS, TNFRSF21, DENND5A }\end{array}$ \\
\hline \multicolumn{4}{|l|}{ Tissue repair } \\
\hline TGF_BETA_SIGNALING & $\begin{array}{l}\text { Genes upregulated in response } \\
\text { to TCFB1 }\end{array}$ & 0.03 & $\begin{array}{l}\text { MAP3K7, PMEPA1, ACVR1, NCOR2, CDK9, SLC20A1, ARID4B, } \\
\text { IFNGR2, RHOA, SERPINE1, WWTR1, FURIN, TRIM33, THBS1, } \\
\text { ID1, TGFB1, JUNB, FKBP1A, SMAD7, KLF10 }\end{array}$ \\
\hline APOPTOSIS & $\begin{array}{l}\text { Genes mediating programmed } \\
\text { cell death (apoptosis) by } \\
\text { activation of caspases }\end{array}$ & 0.03 & $\begin{array}{c}\text { HSPB1, ADD1, ANXA1, IFITM3, PMAIP1, EBP, CASP8, CREBBP, } \\
\text { GADD45A, HMGB2, MADD, PPT1, PRF1, BCL10, RHOT2, } \\
\text { TIMP2, SLC20A1, ATF3, EGR3, IGF2R }\end{array}$ \\
\hline UV_RESPONSE_DN & $\begin{array}{l}\text { Cenes downregulated in } \\
\text { response to ultraviolet radiation }\end{array}$ & 0.03 & $\begin{array}{c}\text { LPAR1, SYNJ2, BHLHE40, NEK7, PLPP3, DMAC2L, LTBP1, } \\
\text { ANXA2, ACVR2A, NFKB1, COL1A1, DDAH1, FBLN5, ITCB3, } \\
\text { MAP2K5, WDR37, DUSP1, PTEN, RGS4, GJA1 }\end{array}$ \\
\hline KRAS_SIGNALING_UP & $\begin{array}{l}\text { Genes upregulated by KRAS } \\
\text { activation }\end{array}$ & 0.02 & $\begin{array}{c}\text { RELN, RGS16, MALL, DOCK2, ANXA10, HDAC9, CD37, EVI5, } \\
\text { INHBA, SERPINA3, SCG3, ANGPTL4, DCBLD2, MAFB, F2RL1, } \\
\text { BTBD3, PPBP, CCL20, LAT2, CBR4 }\end{array}$ \\
\hline MITOTIC_SPINDLE & $\begin{array}{l}\text { Genes important for mitotic } \\
\text { spindle assembly }\end{array}$ & 0.03 & $\begin{array}{c}\text { SORBS2, CCNB2, CD2AP, NEDD9, RICTOR, HOOK3, CENPJ, } \\
\text { ROCK1, CEP192, PKD2, VCL, EPB41, CTTN, CAPZB, CDK1, } \\
\text { NEK2, BIRC5, CLASP1, KIF11, BRCA2 }\end{array}$ \\
\hline E2F_TARGETS & $\begin{array}{l}\text { Genes encoding cell cycle-related } \\
\text { targets of E2F transcription } \\
\text { factors }\end{array}$ & 0.03 & $\begin{array}{c}\text { TBRG4, DNMT1, CDK1, PRKDC, BIRC5, NBN, BRCA2, STAC1, } \\
\text { UBE2T, MCM3, MSH2, PPP1R8, RAD51AP1, HMGB2, BUB1B, } \\
\text { POLD1, RNASEH2A, CDKN3, EZH2, RPA3 }\end{array}$ \\
\hline G2M_CHECKPOINT & $\begin{array}{l}\text { Genes involved in the } \mathrm{G} 2 / \mathrm{M} \\
\text { checkpoint, as in progression } \\
\text { through cell division cycle }\end{array}$ & 0.03 & $\begin{array}{c}\text { EWSR1, CDK1, CUL5, NEK2, BIRC5, KIF11, BRCA2, STAG1, } \\
\text { MCM3, E2F2, CCNF, INCENP, CDKN3, CDC6, EZH2, PAFAH1B1, } \\
\text { E2F1, NASP, JPT1, TMPO }\end{array}$ \\
\hline
\end{tabular}

a critical switch required for EMT, along with VIM and $C D H 2$ encoding key mesenchymal markers. Last, genes that contribute to apoptosis, such as PMAIP1, CASP8, and BCL10, and genes that are important for transforming growth factor- $\beta$ (TGF- $\beta$ ) signaling, such as $R H O A$, TGFB1, and TGFBR1, were included within the leading edges.

Cell division. Four hallmark gene sets involved in cell division were differentially modulated by tesamorelin versus placebo: G2M_CHECKPOINT (NES $=-1.60$, FDR $q$ value $=0.03)$, E2F_TARGETS $(\mathrm{NES}=-1.57$, FDR $q$ value $=0.03)$, MITOTIC_SPINDLE $(\mathrm{NES}=-1.56$, FDR $q$ value $=0.03)$, and 


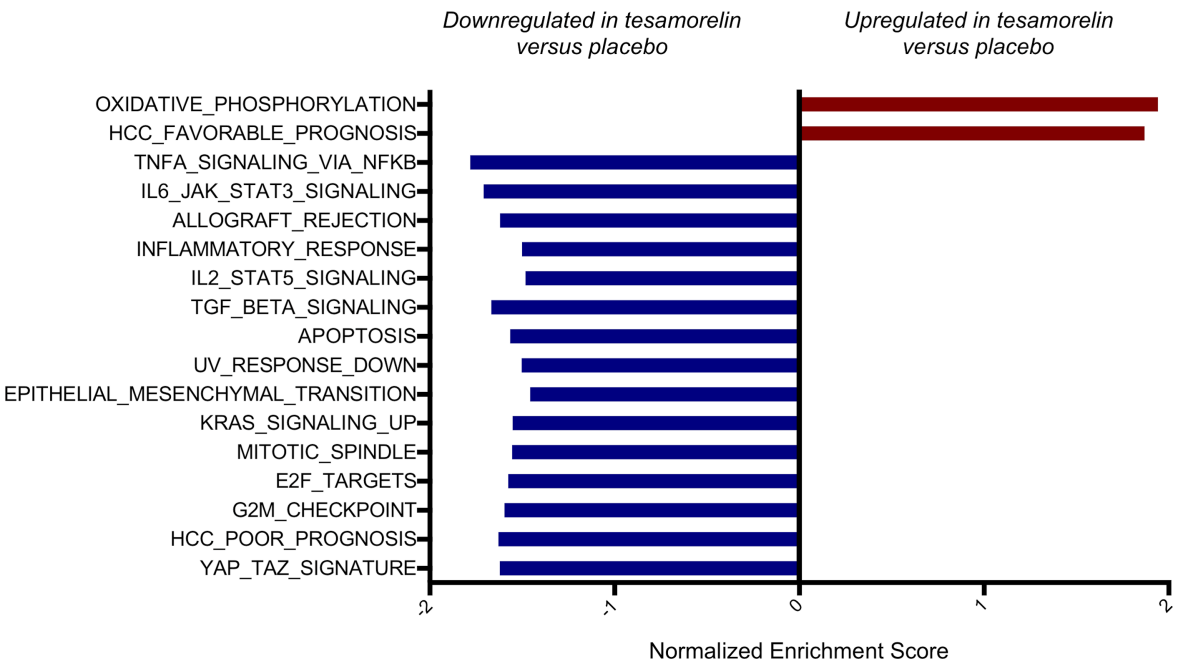

Figure 1. Hepatic gene expression pathways were differentially modulated by tesamorelin versus placebo. A bar graph depicts the normalized enrichment score for hallmark and curated gene sets whose hepatic expression was differentially regulated by tesamorelin versus placebo. Two gene sets pertaining to oxidative phosphorylation and favorable prognosis of hepatocellular carcinoma (HCC) were found to be upregulated in tesamorelin- versus placebo-treated participants. Fifteen gene sets related to inflammation, tissue repair, cell turnover, and poor prognosis of HCC were found to be downregulated in tesamorelin- versus placebo-treated participants. For each pathway shown, the difference between groups had FDR $q$ value less than 0.05 .

KRAS_SIGNALING_UP $($ NES $=-1.55$, FDR $q$ value $=0.02)$. Overall, these gene sets were downregulated among tesamorelin-treated participants and upregulated among placebo-treated participants (Figure 4). The leading edges of these gene sets include genes responsible for DNA replication, including the minichromosome maintenance genes MCM6 and MCM2 and the DNA polymerase POLD1. Also prominent within these leading edges were genes important for mitotic chromosomal segregation, such as CENPJ and CEP192 involved in centrosome function, CLASP1 and NUMA1 involved in mitotic spindle formation, and STAG1 and SMC1A required for cohesion of sister chromatids. Last, genes that encode positive cell cycle regulators, including $C C N B 2, C D K 1, E 2 F 2$, and $E 2 F 1$, as well as the marker of cell proliferation $M K I 67$, were also represented among the leading edges of these gene sets, indicating their relative downregulation among the tesamorelin group.

\section{Effects of tesamorelin on curated gene sets prognostic of HCC}

We next examined curated gene sets that were prognostic of HCC to test the hypothesis that tesamorelin would confer a favorable gene expression profile in this regard. We found that tesamorelin relative to placebo led to upregulation of genes associated with favorable HCC prognosis (NES $=1.87$, FDR $q$ value $=$ 0.0003) (Table 3 and Figure 5). Contained within the leading edge of this gene set were genes responsible for hepatic homeostatic functions. Examples included CYP7A1, SLC10A1, and BAAT central to bile acid metabolism; PON1 and CYP2C9 important for detoxification of xenobiotic compounds; $A F M, R B P 4$, and $G C$ that encode transport proteins; and $A P O H$ and $A P O C 1$ involved in lipoprotein metabolism.

Conversely, tesamorelin was found to downregulate a hepatic stellate cell (HSC) gene signature predictive of poor HCC prognosis (NES $=-1.63$, FDR $q$ value $=0.03)($ Table 3 and Figure 5). Genes included within the leading edge of this gene set were involved in collagen biosynthesis and assembly (e.g., COL5A2, LOXL2), organization of the actin cytoskeleton (e.g., TAGLN, TLN2, SDC3), and growth factor signaling (e.g., PDGFRB, HGF, PDGFRA). Using a curated gene set, we further found that YAP/TAZ signaling, which is implicated in hepatic fibrogenesis and carcinogenesis (6), was downregulated among tesamorelin- versus placebo-treated participants over the 1-year study period (NES $=-1.62$, FDR $q$ value $=0.03)$ (Table 3 ).

Relationships of changes in hepatic gene expression with change in hepatic fat

For each differentially regulated gene set, we assessed whether change in gene expression related to change in hepatic fat fraction. In this regard, we compared 3 groups of participants: placebo-treated individuals, tesamorelin-treated individuals with less than $30 \%$ relative hepatic fat reduction, and tesamorelin-treated 
A
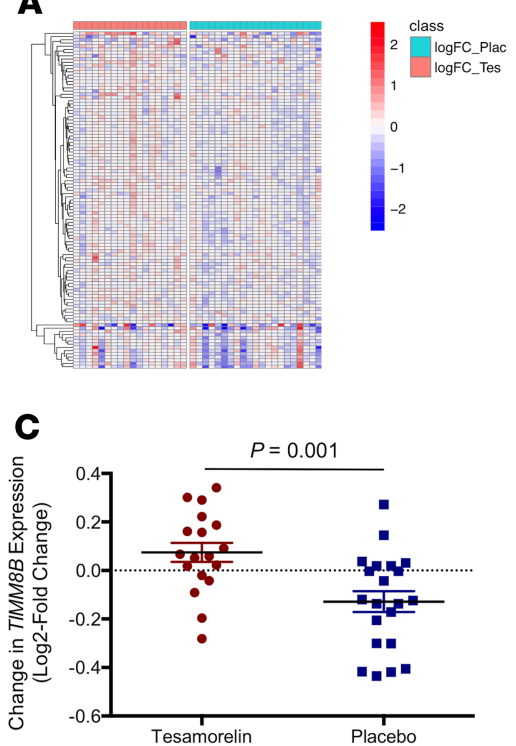

B
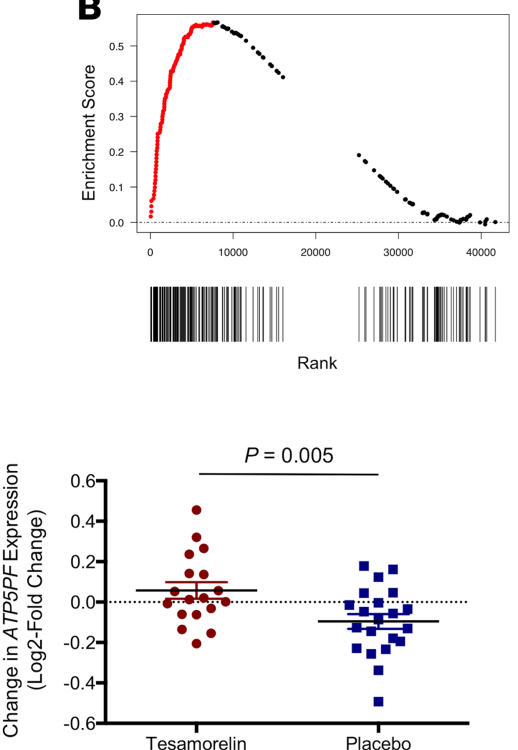

Figure 2. Tesamorelin mediated hepatic upregulation of the oxidative phosphorylation hallmark gene set. (A) Heatmap of $\log _{2}$ fold changes in hepatic gene expression for the hallmark OXIDATIVE_PHOSPHORYLATION gene set. Columns correspond to individual participants; rows represent $\log _{2}$ fold change of individual leading edge genes. There was overall upregulation of oxidative phosphorylation genes among tesamorelin-treated participants (left) and downregulation of oxidative phosphorylation genes among placebo-treated participants (right). (B) CSEA enrichment plot for the hallmark OXIDATIVE_PHOSPHORYLATION gene set. The position of each gene in the gene set in the ranked transcriptome is indicated by a black bar below the plot. Clustering of genes to the left on the ranked list indicates greater upregulation in tesamorelin relative to placebo. Genes corresponding to the leading edge are shown in red. (C) Dot plots of changes in hepatic gene expression for select genes within the OXIDATIVE_PHOSPHORYLATION gene set. Compared with placebo-treated patients $(N=21)$, tesamorelin-treated participants $(N=18)$ exhibited hepatic upregulation of TIMM8B, which is responsible for guiding membrane-spanning proteins into the mitochondrial inner membrane. Individuals assigned to tesamorelin also demonstrated enhanced expression of ATP5PF, which encodes a subunit of the mitochondrial ATP synthase (complex V). $P$ values were corrected for multiple testing and calculated using negative binomial generalized linear models. Error bars correspond to mean and standard error of the mean.

individuals with at least $30 \%$ relative hepatic fat reduction. Across these groups, consistent and significant trends were observed, demonstrating greater change in gene expression with a stepwise change in hepatic fat fraction from placebo- to tesamorelin-treated group with less than $30 \%$ fat reduction to tesamorelin-treated group with at least $30 \%$ fat reduction (i.e., greater change in gene expression with greater reduction in hepatic fat, Supplemental Table 2). No significant relationship was found between treatment status and the expression of de novo lipogenesis genes as assessed by Gene Ontology pathways.

\section{Relationships of changes in hepatic gene expression with change in fibrosis-related gene score and IGF1 transcript levels}

Finally, among tesamorelin-treated participants, we assessed relationships between changes in hepatic expression of differentially regulated gene sets and change in fibrosis-related gene score based on the hepatic expression of 18 genes previously shown to correlate with fibrosis (7). Among our overall sample at baseline, we found a strong association between fibrosis-related gene score and histologic fibrosis stage $(P=0.0009$; post-ANOVA test for linear trend $P=0.0001$ ), which validated our use of this gene set as a proxy for hepatic fibrosis (Supplemental Figure 2). Of note, we demonstrated that hepatic upregulation of genes involved in oxidative phosphorylation and favorable HCC prognosis were associated with decreased fibrosis-related gene score. Moreover, hepatic downregulation of genes involved in inflammation, tissue repair, cell division, and unfavorable HCC prognosis also strongly correlated with decreased fibrosis-related gene score (Supplemental Table 3 and Figure 6).

Within the tesamorelin group, we also found that changes in hepatic IGF1 transcript levels were associated with changes in pathways involved in tissue repair and carcinogenesis, including TGF_BETA_ SIGNALING, UV_RESPONSE_DN, and YAP_TAZ_SIGNATURE. For each relationship, a greater rise in IGF1 transcript levels was associated with more pronounced downregulation of these pathways (Supplemental Table 3, Supplemental Figure 3). 


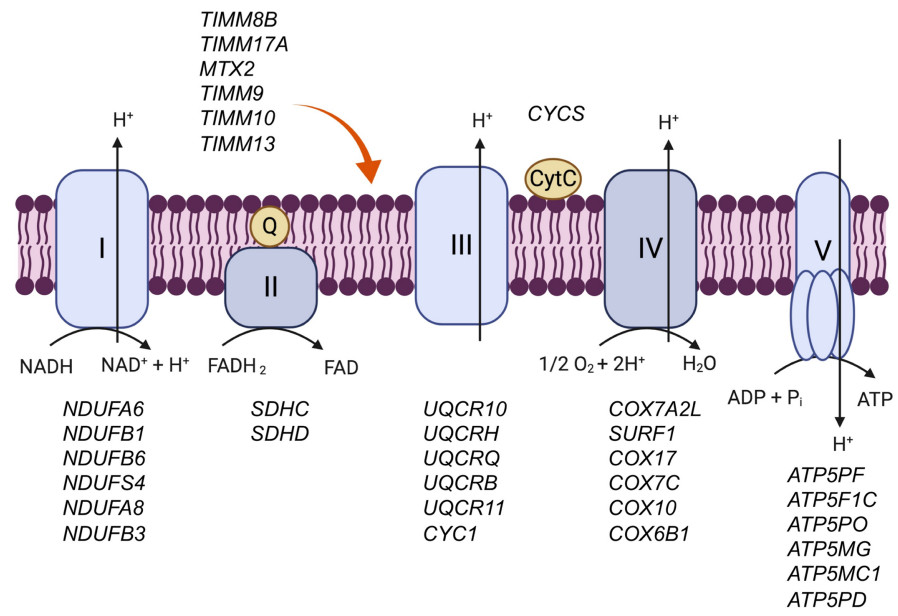

Figure 3. Oxidative phosphorylation genes upregulated by tesamorelin included genes involved in the electron transport chain. Select genes within the leading edge of the OXIDATIVE_PHOSPHORYLATION gene set are listed beside the complex to which they correspond. Tesamorelin was found to modulate genes responsible for the structure and function of all 5 complexes within the electron transport chain.

\section{Discussion}

In HIV-associated NAFLD, we showed that tesamorelin, relative to placebo, increased hepatic expression of genes important for oxidative phosphorylation and decreased expression of genes involved in inflammation, tissue repair, and cell division. Furthermore, we found that treatment with tesamorelin led to reciprocal up- and downregulation of genes associated with favorable and poor HCC prognosis, respectively. Notably, these changes in hepatic gene expression correlated with improved fibrosis-related gene score among tesamorelin-treated participants. Taken together, our findings inform our knowledge of the biology of pulsatile GH action and provide a potential mechanistic basis for the observed clinical effects of tesamorelin on the liver.

In an unbiased analysis of the MSigDB hallmark gene sets, we found that tesamorelin led to hepatic upregulation of oxidative phosphorylation genes compared with placebo over 1 year. Furthermore, among tesamorelin-treated participants, enhanced expression of these genes related to oxidative phosphorylation was associated with decreased fibrosis-related gene score and degree of hepatic fat reduction using a clinically defined $30 \%$ stratification across treatment groups (8). Moreover, increases in oxidative phosphorylation were related to increased IGF-1 transcription, providing evidence linking augmented GH signaling to increased oxidative phosphorylation. Mitochondria play a key role in fatty acid catabolism, and dysfunction in this key organelle has been implicated as a key feature in NAFLD pathogenesis (9). In a NASH rat model, declines in hepatic oxidative phosphorylation efficiency, electron transport chain enzyme activities, and mitochondrial transmembrane potential were seen as hepatic steatosis progressed (10). Similarly, liver biopsy specimens obtained from patients with NASH were found to have reduced maximal mitochondrial respiration and blunted expression of transcription factors regulating mitochondrial biogenesis and the electron transport chain versus controls (11). Mitochondrial impairment may promote hepatic fat accumulation and generation of toxic lipid metabolites, increasing oxidative stress, cell death, inflammation, and fibrosis, which are key events in NAFLD progression (9). Strategies to restore oxidative phosphorylation may be useful to ameliorate the clinical course of NAFLD progression (12).

Our finding that tesamorelin upregulated expression of hepatic oxidative phosphorylation genes is supported by prior work showing that $\mathrm{GH}$ axis augmentation has beneficial mitochondrial effects. In a small study of healthy men and women, short-term infusion of GH acutely elevated skeletal muscle mitochondrial oxidative capacity, heightened the abundance of muscle mRNAs encoding mitochondrial proteins, and shifted whole-body substrate utilization toward fat oxidation (13). Similarly, in a rat model of cirrhosis, treatment with IGF-1 was demonstrated to increase mitochondrial membrane potential and ATP synthase activity and to reduce intramitochondrial free radical production, caspase activation, and apoptosis (14). Last, in obese adults treated with tesamorelin, we previously found an association between increased serum IGF-1 and accelerated ATP-dependent phosphocreatine recovery rate in skeletal muscle 
A

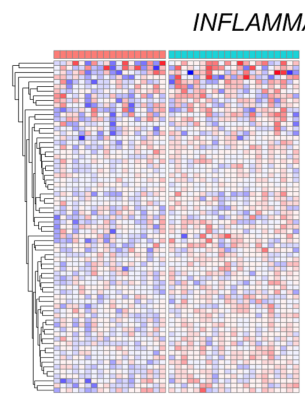

NFLAMMATORY_RESPONSE

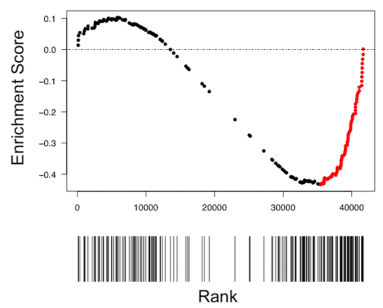

\section{Inflammation}

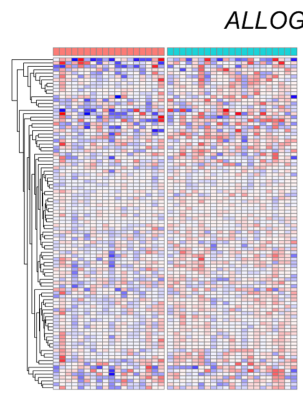

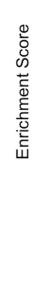
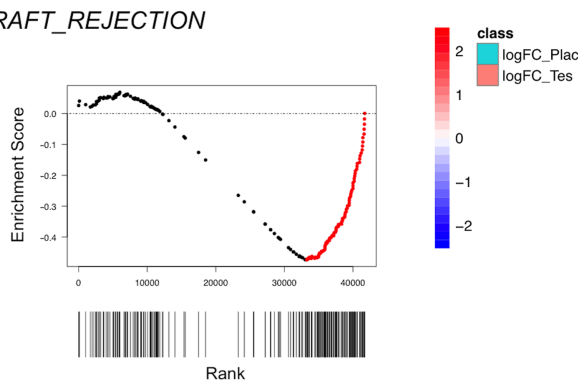

B

Tissue Repair

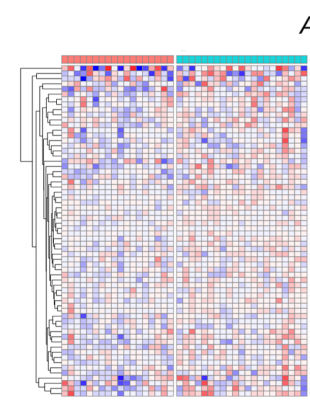

APOPTOSIS
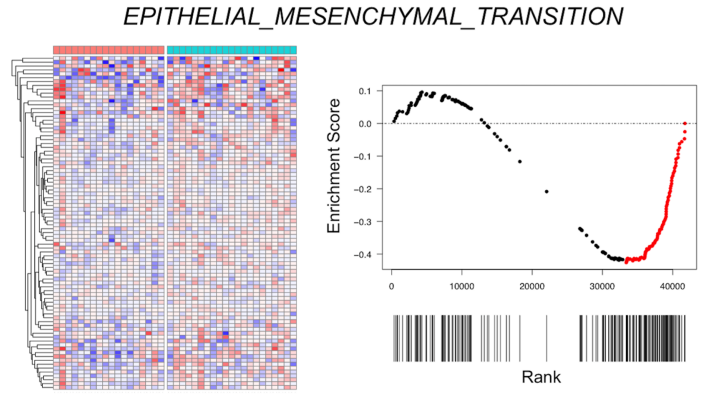

Cell Division

C

G2M_CHECKPOINT
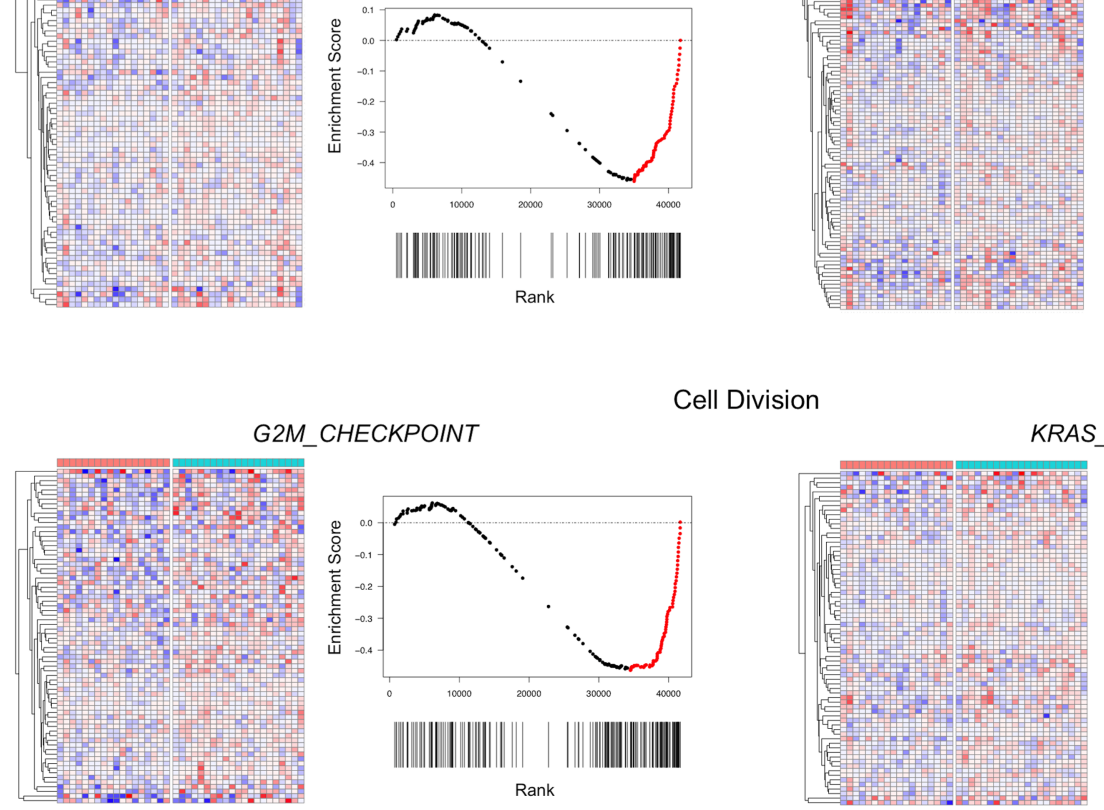

KRAS_SIGNALING_UP

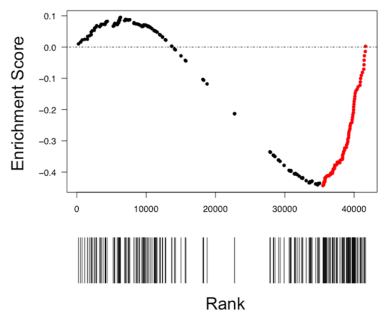

Figure 4. Tesamorelin led to hepatic downregulation of hallmark gene sets involved in inflammation, tissue repair, and cell division. Heatmaps and enrichment plots demonstrate differential changes in hepatic expression of select gene sets involved in (A) inflammation, (B) tissue repair, and (C) cell turnover by treatment status. For the heatmaps, columns correspond to individual participants, whereas rows represent log fold change of individual leading edge genes. Overall, these pathways were downregulated among tesamorelin-treated participants (left) and upregulated among placebo-treated participants (right). Enrichment plots similarly show that genes within the featured gene sets were overrepresented at the bottom of the entire ranked list. Genes corresponding to the leading edge are shown in red.

following exercise (15). Overall, improved mitochondrial function in response to enhanced GH secretion may be a key strategy whereby tesamorelin attenuates NAFLD severity in HIV.

Compared with placebo, we also showed that tesamorelin led to robust downregulation of key inflammatory gene pathways involved in the innate and adaptive immune response. Notable among the gene sets differentially regulated between groups were those involved in tumor necrosis factor- $\alpha$ (TNF- $\alpha$ ) and interleukin-6 (IL-6) signaling. Specifically, studies have shown that hepatic expression of these cytokines was elevated in patients with NASH in proportion to the degree of inflammation $(16,17)$.

The effects of tesamorelin on multiple inflammatory pathways suggest that augmenting endogenous GH may be effective in reducing hepatic inflammation in HIV-associated NAFLD. Consistent with these findings, prior studies have shown that GH deficiency is a systemic inflammatory state that is attenuated by GH axis augmentation $(18,19)$. Additionally, in a mouse model, liver-specific ablation of the GH receptor led to hepatic steatosis with increased macrophage infiltration and enhanced hepatic expression of cytokines, including TNF- $\alpha$ and IL-6 (20). In our recent clinical trial, change in histologic inflammation was not found to significantly differ between treatment and placebo groups, though sample size was limited. 
Table 3. Curated gene sets modulated by tesamorelin versus placebo

\begin{tabular}{|c|c|c|c|}
\hline Gene set name & Gene set description & FDR q value & Top leading edge genes \\
\hline HCC_POOR_PROGNOSIS & $\begin{array}{c}\text { Gene expression signature specific } \\
\text { to HSCs associated with poor } \\
\text { prognosis in HCC }\end{array}$ & 0.03 & $\begin{array}{l}\text { COL5A2, LOXL2, PDGFRB, TAGLN, } \\
\text { FRZB, BCN, SEMA3A, TLN2, } \\
\text { DCBLD2, TNXB, HGF, ADAMTS2, } \\
\text { TCFB3, TNFRSF11B, RGS7, EDNRB, } \\
\text { SDC3, PDGFRA, ITGA9 }\end{array}$ \\
\hline
\end{tabular}

Nonetheless, tesamorelin-treated individuals with higher histologic inflammation at baseline exhibited a greater decline in inflammatory activity over 1 year (3). In addition, compared with placebo, tesamorelin was demonstrated to reduce systemic immune activation as measured by circulating C-reactive protein levels (3). As such, our gene expression data expand upon the clinical signal that we observed.

In the current study, we also found that decreased hepatic expression of inflammatory gene sets was associated with a reduction in fibrosis-related gene score among tesamorelin-treated participants. Given that NASH is an important precursor to fibrosis, reducing hepatic inflammation may provide a mechanistic basis whereby tesamorelin slows fibrosis progression in HIV-associated NAFLD. Evaluation of specific leading edge genes in the inflammatory gene sets differentially regulated by tesamorelin versus placebo suggests potential pathways that may mediate the fibrogenic response. As 2 examples, leading edge genes C-C motif chemokine ligands 5 (CCL5) and 20 (CCL20) are highly expressed among patients with NAFLD in proportion to the severity of histologic disease $(21,22)$. In vitro treatment of HSCs with CCL5 or CCL20 has been shown to directly induce fibrogenesis $(21,22)$. Moreover, in a recent phase IIb trial of NASH, antagonism of CCL5 receptors with cenicriviroc reduced hepatic fibrosis without worsening steatohepatitis (23). Taken together, downregulation of inflammatory genes that dually promote fibrogenesis may contribute to the relationship that we observed between changes in inflammatory pathways and fibrosis gene signature among tesamorelin-treated participants.

Importantly, we additionally found that tesamorelin led to hepatic downregulation of gene pathways involved in tissue repair, including those related to apoptosis, EMT, and TGF- $\beta$ signaling, compared with placebo. Moreover, these changes in gene expression directly correlated with change in fibrosis-related gene score within the tesamorelin group. While tissue repair responses enable liver regeneration following an acute insult, ongoing activation of these pathways in the setting of chronic injury may lead to hepatic fibrosis (24). As an example, high rates of hepatocyte apoptosis, as has been described in patients with NAFLD, may trigger fibrogenesis (25). Similarly, TGF- $\beta$ signaling is the most prominent pathway driving hepatic fibrogenesis (26). In this regard, TGF- $\beta$ signaling results in activation of HSCs, transdifferentiation of epithelial cells to myofibroblasts, and enhanced production of ECM proteins $(26,27)$. Downregulation of the tissue repair response by tesamorelin may constitute an important process by which this agent slows the clinical progression of liver disease.

Our findings that tesamorelin reduced hepatic expression of genes involved in tissue repair support a role for augmented GH secretion and IGF-1 in blunting fibrogenesis. In this regard, data in the current study demonstrate significant inverse relationships between changes in IGF1 transcript levels and key tissue repair pathways. Relatedly, in both NASH and cirrhotic animal models, augmentation of hepatic IGF-1 signaling has been shown to result in fibrosis regression $(28,29)$. In one such study, IGF-1 treatment led to hepatic upregulation of metalloproteinases responsible for ECM degradation, as well as downregulation 
A

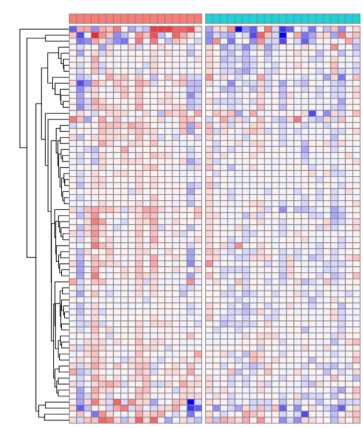

HCC_FAVORABLE_PROGNOSIS

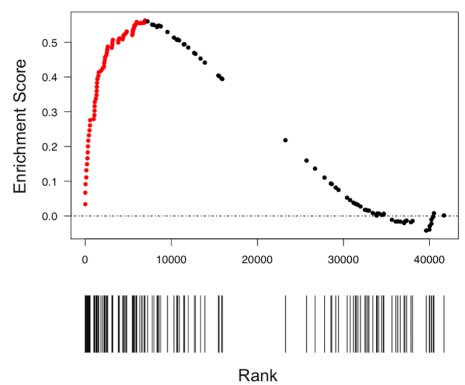

B

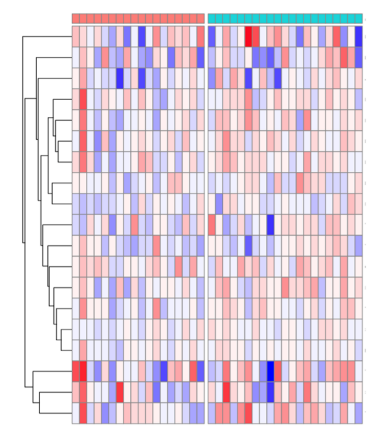

HCC POOR PROGNOSIS
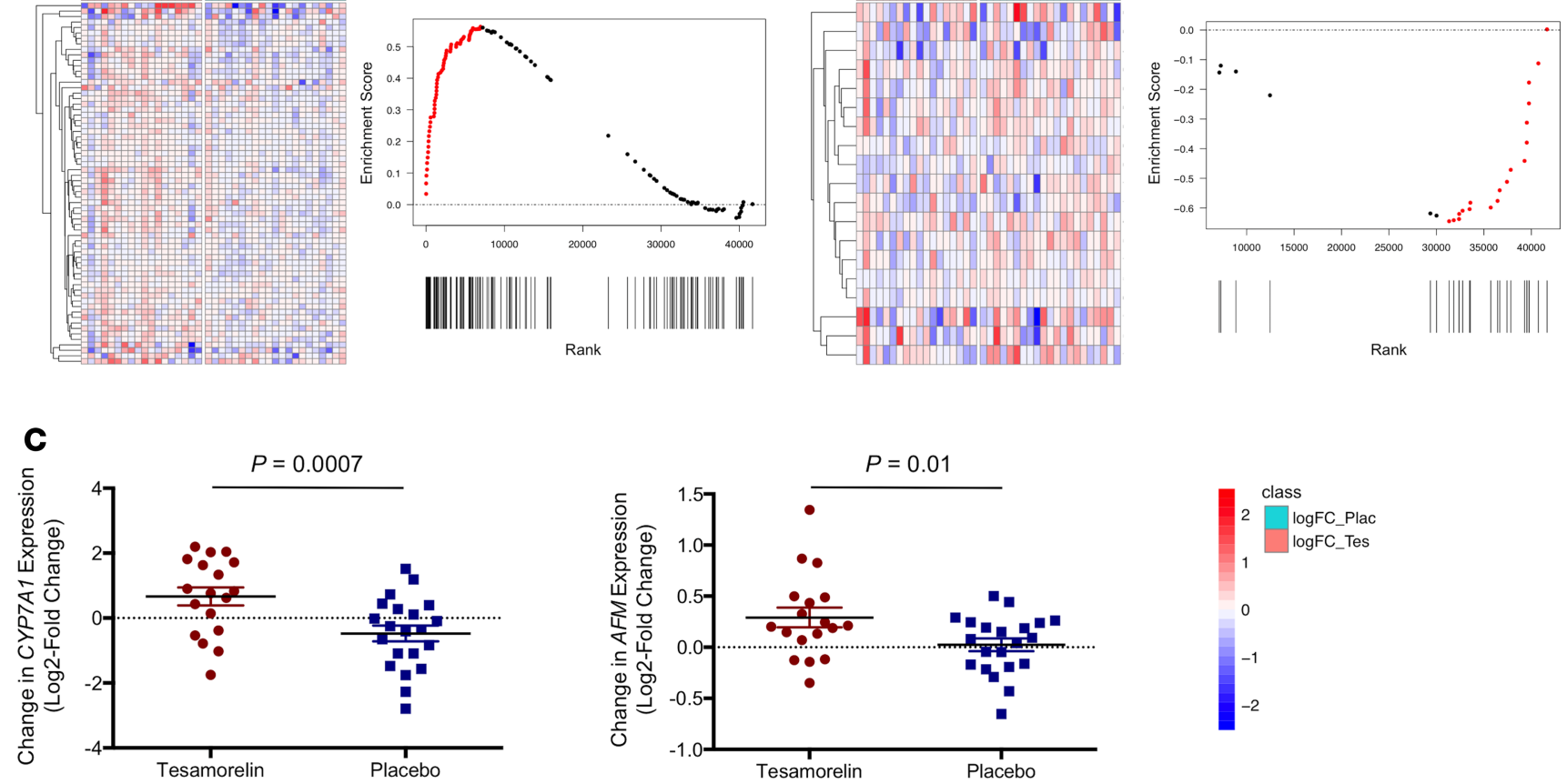

Rank

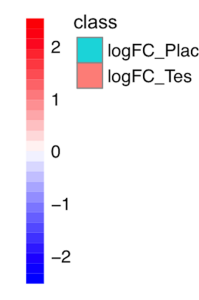

Figure 5. Tesamorelin shifted hepatic gene expression toward a profile associated with a more favorable HCC prognosis. (A) A heatmap and an enrichment plot are shown, depicting changes in hepatic expression of a curated gene set associated with favorable HCC prognosis. In the heatmap, columns correspond to individual participants, whereas rows represent $\log _{2}$ fold change of individual leading edge genes. Genes predictive of a favorable HCC prognosis were overall upregulated among tesamorelin-treated participants (left) and downregulated among placebo-treated participants (right). The enrichment plot likewise demonstrates an overrepresentation of genes associated with favorable prognosis at the top of the entire ranked list. Genes corresponding to the leading edge are shown in red. (B) A heatmap and an enrichment plot demonstrate changes in hepatic expression of a curated gene set associated with poor HCC prognosis. In contrast to our findings with regard to the favorable prognosis gene set, genes linked to a poor HCC prognosis were reciprocally downregulated and upregulated in the tesamorelin and placebo groups, respectively. Furthermore, per the enrichment plot shown, genes within the poor prognosis gene set were overrepresented at the bottom of the entire ranked list. (C) Dot plots of changes in hepatic gene expression for select genes within the favorable prognosis gene set are shown. Compared with placebo-treated patients $(N=21)$, tesamorelin-treated participants $(N=$ 18) were found to have hepatic upregulation of CYP7A1 encoding a critical regulatory enzyme in bile acid biosynthesis and cholesterol homeostasis and AFM encoding a member of the albumin gene family responsible for protein transport. $P$ values were corrected for multiple testing and calculated using negative binomial generalized linear models. Error bars correspond to mean and standard error of the mean.

of the profibrogenic mediator Tgfb and metalloproteinase inhibitors Timp1 and Timp2 (29). Of note, the changes in gene expression in this prior report resembled those that we observed with GHRH analog therapy, which we now extend for the first time to our knowledge to humans. IGF-1 has been demonstrated to decrease apoptosis in multiple cell lines (30) and to induce senescence of HSCs in a p53-dependent manner (28), which may underlie these phenotypic changes.

Tesamorelin downregulated hepatic genes involved in cell division as compared with placebo. Furthermore, reduced expression of these genes among tesamorelin-treated individuals was associated with a decrease in fibrosis-related gene scores. Hepatocyte proliferation has been recognized as a critical step underlying NAFLD pathogenesis even early in the course of the disease (31). In this regard, liver specimens from patients and animal models with NAFLD have consistently shown elevations in markers of cell proliferation, including Ki-67, E2F1, cyclin-dependent kinase 4, and gank (31-33). Importantly, such factors have been implicated in the development of hepatic steatosis and fibrosis. As an example, in $d b / d b$ leptin-deficient mice, E2f1 knockout was found to prevent hepatic steatosis through crosstalk with key metabolic pathways (34). Furthermore, in a mouse model of cirrhosis, E2F1 deficiency was demonstrated to protect against liver fibrosis and associated hepatic dysfunction (33). Beyond accelerating NAFLD progression, high rates of cell division may predispose to HCC. Notably, the minichromosome maintenance genes MCM2 and MCM6, which were both downregulated in tesamorelin-treated participants, were shown to be elevated in HCC in association with poor survival (35).

Tesamorelin reduced fibrosis progression in our clinical trial, an important factor associated with the development of HCC. Although assessment of HCC outcomes was beyond the scope of our trial, we used 
A

Cellular Metabolism

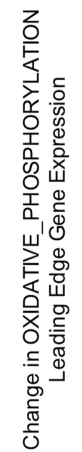

B

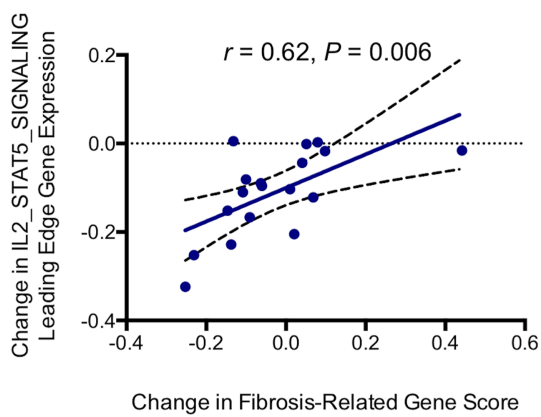

Inflammation

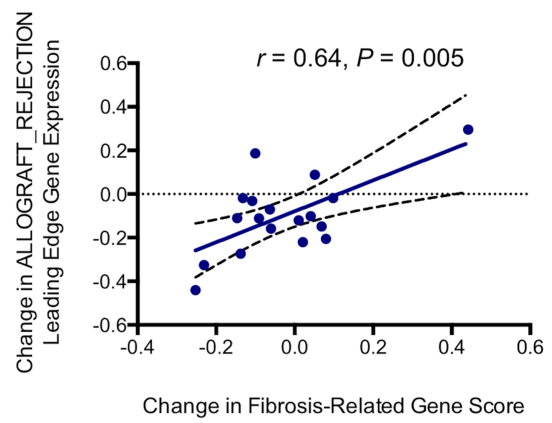

D

Cell Division

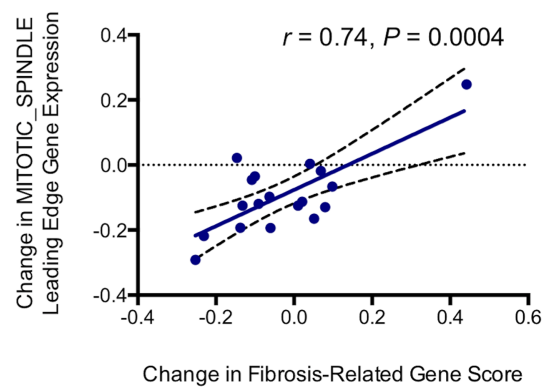

Figure 6. Changes in hepatic expression of hallmark gene sets were correlated with change in mean fibrosis-related gene score in tesamorelin-treated participants. (A) Within the tesamorelin group, a reduction in fibrosis-related gene score was associated with an increase in hepatic expression of oxidative phosphorylation genes. (B-D) In contrast, among tesamorelin-treated participants, change in fibrosis-related gene score was directly associated with changes in hepatic expression of gene sets pertaining to inflammation, tissue repair, and cell proliferation. For all graphs, axes reflect log ${ }_{2}$ fold change in mean leading edge gene expression. Linear regression lines with 95\% confidence intervals are shown, with $r$ and $P$ value from Pearson correlation. $N=18$ for all graphs. Graphs with red and blue dots correspond to pathways up- and downregulated by tesamorelin versus placebo, respectively.

curated gene sets in this analysis to assess potential relationship to HCC risk. We found that tesamorelin upregulated genes associated with a favorable HCC prognosis and downregulated genes associated with poor HCC prognosis. Tesamorelin also downregulated the YAP/TAZ signaling pathway, which is a key driver of fibrosis and cancer (6). This favorable modulation of cancer-related gene sets parallels the widespread improvement in metabolic, inflammatory, fibrogenic, and proliferative pathways that we observed in the current analysis. Importantly, these changes also run counter to theoretical concerns that increased GH/IGF-1 signaling may exacerbate cancer risk, which have been raised in some contexts. In this regard, it is important to consider that GHRH analog therapy, unlike GH, augments physiologic GH pulsatility with gains in IGF-1 that remain within the normal range.

To our knowledge, this is the first study in humans to investigate the mechanistic underpinnings for a NAFLD therapy using a whole-transcriptome approach. Additionally, study participants comprised a modern cohort of PLWH, and the results derive from a 1-year randomized controlled trial with a placebo comparator. Given that the HIV population is at high risk of NAFLD and increased fibrosis progression rates $(1,2,36)$, there is a critical need for dedicated studies among this patient group. Our findings also may yield insights for other populations with NAFLD and thus provide a strong rationale for additional studies. Limitations of our analysis include its relatively small sample size. Moreover, although the sample was a subset of our larger study cohort, we do not believe there were any factors systematically contributing to selection into the subset, as supported by the overall similarities between this study cohort and the entire cohort. Because we have tissue from only 2 time points, we cannot determine whether changes in gene expression are a direct effect of tesamorelin or are mediated by other biological effects indirectly resulting from tesamorelin. Thus, although we saw significant correlations between increased IGF-1 transcription 
and changes in gene expression, further studies are needed to assess whether these effects are directly related to augmented GH signaling. Though we did not see effects over time of tesamorelin to downregulate de novo lipogenesis gene pathways, our assessments were made in the fasting state, when lipogenesis is low. Additionally, GH axis augmentation may result in modulation of pathways at the protein translational or posttranslational level that would evade detection at a transcriptome level. Relatedly, a change in gene expression does not necessarily correspond to changes in tissue function.

In summary, among individuals with HIV-associated NAFLD, GH axis augmentation with tesamorelin led to changes in hepatic gene expression that reflect an overall return toward liver health. We found that tesamorelin upregulated oxidative phosphorylation genes and downregulated genes involved in inflammation, tissue repair, and cell turnover compared with placebo. Further, tesamorelin shifted hepatic gene expression toward a profile associated with a favorable HCC prognosis. By expanding our knowledge of the effects of $\mathrm{GH}$ axis augmentation on hepatic biology, our findings raise the possibility of novel clinical benefits of this strategy in the treatment of NASH that should be evaluated in future large-scale efforts.

\section{Methods}

Study design. We previously conducted a randomized, double-blind trial in which individuals with HIV-associated NAFLD were assigned to receive the GHRH analog tesamorelin $2 \mathrm{mg}$ daily or identical placebo for 12 months (3). The current analysis leveraged liver biopsy specimens from this recent trial to identify gene pathways that were differentially modulated by treatment and to investigate associations of changes in gene expression with changes in clinical outcome among tesamorelin-treated individuals. These findings have not been previously reported.

We enrolled 61 men and women 18-70 years old who had documented HIV infection as well as hepatic steatosis as defined by liver fat fraction at least $5 \%$ on ${ }^{1} \mathrm{H}$-magnetic resonance spectroscopy $\left({ }^{1} \mathrm{H}-\mathrm{MRS}\right)$. Participants were required to have been on stable ART for at least 3 months with $\mathrm{CD} 4^{+} \mathrm{T}$ cell count greater than 100 cells $/ \mathrm{mm}^{3}$ and HIV viral load less than 400 copies $/ \mathrm{mL}$. Exclusion criteria included excess alcohol use ( $>20 \mathrm{~g}$ daily for women or $>30 \mathrm{~g}$ daily for men), active hepatitis B or C as previously described (3), other known hepatic disease, cirrhosis, and inadequately controlled diabetes mellitus (HbA1c $\geq 7 \%$ ). Participants were enrolled at the Massachusetts General Hospital (MGH, Boston, Massachusetts) and the NIH (Bethesda, Maryland) between August 20, 2015, and January 16, 2019.

Study procedures. All study procedures were conducted in a fasting state. Hepatic ${ }^{1} \mathrm{H}-\mathrm{MRS}$ was performed for measurement of hepatic fat fraction. Baseline evaluation also included an ultrasound-guided percutaneous liver biopsy yielding 2 cores, which was completed on all participants except for those with a contraindication (e.g., anticoagulation). The first core was fixed in formalin and subsequently underwent histopathologic review by a single expert pathologist blinded to treatment. Histologic scoring, including NAFLD Activity Score and fibrosis stage, was performed according to the Nonalcoholic Steatohepatitis Clinical Research Network scoring system (37). The second core was placed in an RNA stabilization reagent (RNAlater, QIAGEN) and stored at $-80^{\circ} \mathrm{C}$ for gene expression analysis. Both ${ }^{1} \mathrm{H}-\mathrm{MRS}$ and liver biopsy were repeated at 12 months following randomization.

cDNA library construction. Following extraction from liver tissue using RNeasy Plus Mini Kit (QIAGEN), total RNA was quantified using the Quant-iT RiboGreen RNA Assay Kit (Thermo Fisher Scientific) and normalized to $5 \mathrm{ng} / \mu \mathrm{L}$. Following plating, $2 \mu \mathrm{L}$ of External RNA Controls Consortium controls (using a 1:1000 dilution) and a k562 control were spiked into each sample. A 200 ng aliquot of each sample was taken for library preparation, using Illumina TruSeq Stranded mRNA Sample Preparation Kit. Oligo-dT beads were used to select mRNA from the total RNA sample, followed by heat fragmentation and cDNA synthesis from the RNA template. The resultant 400 bp cDNA underwent dual-indexed library preparation: "A" base addition, adapter ligation using P7 adapters, and PCR enrichment using P5 adapters. After enrichment the libraries were quantified using Quant-iT PicoGreen (Thermo Fisher Scientific, 1:200 dilution).

Illumina sequencing. Pooled libraries were normalized to $2 \mathrm{nM}$ and denatured using $0.1 \mathrm{M} \mathrm{NaOH}$ before sequencing. Flow cell cluster amplification and sequencing were performed according to the manufacturer's protocols using the NovaSeq S2 (Illumina) to produce $101 \mathrm{bp}$ paired-end reads with 8-base index barcodes. Data were analyzed using the Broad Picard Pipeline, which includes demultiplexing and data aggregation.

Alignment and quality control. All samples were analyzed using the bcbio-nextgen RNA-Seq analysis pipeline (https://bcbionextgen.readthedocs.org/en/latest/). BAM files (converted back to FASTQ read files) were examined for quality issues using FastQC (http://www.bioinformatics.babraham.ac.uk/projects/fastqc/) to 
ensure library generation and sequencing were suitable for further analysis. Reads were aligned to UCSC build hg38 of the human genome (Homo sapiens), augmented with transcript information from Ensembl release 94 using hisat2 (38). Alignments were analyzed for evenness of coverage, rRNA content, genomic context (for example, alignments in known transcripts and introns), complexity, and other quality checks using a combination of FastQC, Qualimap (39), MultiQC (https://github.com/ewels/MultiQC), and custom tools. Counts of reads aligning to known genes were generated by featureCounts (40) and used as input for principal components analysis and hierarchical clustering to identify possible outliers. Additional patterns of gene expression were visualized using the DEGReport Bioconductor package (41).

A total of 19 participants randomized to tesamorelin and 24 participants randomized to placebo had paired liver specimens available for gene sequencing. Four participants, 1 assigned to tesamorelin and 3 to placebo, were excluded from subsequent analyses because of poor RNA samples, resulting in a total of 18 participants randomized to tesamorelin and 21 randomized to placebo with RNA-Seq data included in our analysis. A comparison between characteristics of the participants included in this study and the entire study cohort is shown in Supplemental Table 1. The RNA-Seq data were submitted to the Gene Expression Omnibus repository at the National Center for Biotechnology Information (accession number GSE150026).

Gene set enrichment analysis. To identify pathways differentially modulated from pre- to posttreatment time points between tesamorelin- and placebo-treated participants, GSEA was performed using the desktop module from the Broad Institute (www.broadinstitute.org/gsea/). First, transcripts per million measurements per isoform were generated by quasialignment using Salmon (42). Differential expression at the gene level was called with DESeq2 (43) using counts per gene estimated from the Salmon quasialignments by tximport (44). To determine changes in gene expression over time between treatment groups, we used a repeated-measures model that controlled for subject-specific effects, such as sex, and used an interaction term of treatment $\times$ time point.

Next, transcripts were ranked between tesamorelin- and placebo-treated participants, using the DESeq2 test statistic (Wald's test). Transcripts with greater upregulation from baseline in tesamorelin-treated participants relative to placebo were ranked at the top of the list, and those with greater downregulation in tesamorelin-treated participants relative to placebo at the bottom of the list. GSEA was performed on the ranked transcript list using 1000 gene set permutations and random seeding. GSEA leading edge genes are the subset of genes in a significantly enriched gene set that account for the enrichment signal and were used for subsequent quantification of pathway gene expression. Heatmaps of leading edge gene $\log _{2}$ fold change expression levels from pretreatment to posttreatment time points were generated with pheatmap (45). Transcripts were organized on the $y$ axis by unsupervised hierarchical clustering (Euclidean distance, complete linkage), and samples were manually organized by treatment on the $x$ axis. Gene sets with FDR $<0.05$ were considered enriched.

Gene sets used included the MSigDB hallmark gene set collection (4) and custom gene sets. We examined a custom set of genes associated with a favorable prognosis in HCC. To develop this set, we searched the Human Protein Atlas for genes expressed in hepatic tissue that were associated with a favorable prognosis in HCC (46). The resulting gene list was filtered to exclude genes previously described in a prognostic liver signature (47) to produce our HCC_FAVORABLE_PROGNOSIS gene set. Furthermore, we derived a gene set predictive of poor HCC prognosis from a subset of HSC signature genes that have been previously correlated with poor HCC outcome (HCC_POOR_PROGNOSIS) (48). Relatedly, to study a key pathway involved in carcinogenesis, we leveraged a published set of YAP/TAZ signature genes that had been characterized through in silico meta-analyses of Hippo signaling modules in cancer (YAP_TAZ_SIGNATURE) (49).

Correlation analyses. DESeq2 variance stabilizing-transformed data were used to calculate the $\log _{2}$ fold change in gene expression between pretreatment and posttreatment time points. Mean values for leading edge gene $\log _{2}$ fold change were calculated for each gene set on each subject.

Similarly, for each subject, we calculated a fibrosis-related gene score as the mean $\log _{2}$ fold change for a set of 18 genes (FIBROSIS_SIGNATURE) that was previously identified to be predictive of fibrosis stage in an analysis of patients with NAFLD (7).

Statistics. Differential gene expression between tesamorelin- and placebo-treated patients was calculated using negative binomial generalized linear models (DESeq2). We assessed the relationship between mean fibrosis-related gene score and histologic fibrosis stage among our subjects at baseline using a 1-way ANOVA including a test for linear trend (GraphPad Prism). Pearson correlation was then used to compare the change in mean leading edge gene transcript abundance to the change in mean transcript abundance of the 
FIBROSIS_SIGNATURE set among the tesamorelin group. Additional comparisons were made of mean leading edge gene fold change expression values with fold change in IGF1 expression. In addition, changes in mean leading edge gene expression were assessed in relationship to responsiveness to tesamorelin as defined by a clinically significant reduction in hepatic fat fraction that has been previously established (8). In this regard, we compared changes in gene expression among 3 groups: placebo-treated individuals, tesamorelin-treated individuals with less than $30 \%$ relative hepatic fat reduction, and tesamorelin-treated individuals with at least 30\% relative hepatic fat reduction, using 1-way ANOVA for overall effects and linear trend analysis. Statistical tests with $P<0.05$ were considered statistically significant. We used the Benjamini-Hochberg procedure when correcting for multiple tests.

Study approval. Informed consent in writing was obtained from each participant. The institutional review boards at MGH and the NIH approved this study.

\section{Author contributions}

LTF contributed to the conduct of the study, conception and execution of the analyses presented, and preparation of the manuscript. JMB contributed to the conception and execution of the analyses presented and preparation of the manuscript. GA contributed to the conception and execution of the analyses presented and preparation of the manuscript. SJHS contributed to the conception and execution of the analyses presented and preparation of the manuscript. MNF contributed to the conduct of the study and editing of the manuscript. JP contributed to the conduct of the study and editing of the manuscript. IZ contributed to the conduct of the study and editing of the manuscript. CSP contributed to the conduct of the study and editing of the manuscript. KEC contributed to the conduct of the study and editing of the manuscript. MT contributed to the conduct of the study and editing of the manuscript. DEK contributed to the conduct of the study, histopathologic review, and editing of the manuscript. $\mathrm{CMH}$ contributed to the design and conduct of the study, the conception of the analyses presented, and editing of the manuscript. TLS contributed to the design and conduct of the study and preparation and editing of the manuscript. RTC contributed to the conception and execution of the analyses presented and preparation of the manuscript. SKG contributed to the design and conduct of the study, conception and execution of the analyses presented, and preparation and editing of the manuscript.

\section{Acknowledgments}

We would like to acknowledge and thank the research volunteers who participated in this study as well as the Clinical Research Center staff at the MGH and the NIH. This study was supported by NIH grants U01AI115711 (CMH and SKG), P30DK040561 (SKG), K23HD100266 (LTF), 1KL2TR002542 (LTF), P30AI06035 (JMB and SHJS), R01DK108370 (RTC), R01AI136715 (RTC), U19 AI082630 (RTC), and MGH Research Scholars Program (RTC); in part by the National Institute of Allergy and Infectious Disease Intramural Research Program; and in part by the Intramural Research Program of the NIH, National Cancer Institute. The study drug was provided at reduced cost by Theratechnologies, Inc., which had no role in the design or conduct of the study, analysis of the data, or preparation of this manuscript.

Address correspondence to: Steven K. Grinspoon or Raymond T. Chung, Massachusetts General Hospital, 55 Fruit Street 5LON207 (SKG) or Warren 1007 (RTC), Boston, Massachusetts 02114, USA. Phone: 617.724.9109; Email: sgrinspoon@mgh.harvard.edu (SKG). Phone: 617.724.7562; Email: rtchung@mgh. harvard.edu (RTC).

1. Maurice JB, Patel A, Scott AJ, Patel K, Thursz M, Lemoine M. Prevalence and risk factors of nonalcoholic fatty liver disease in HIV-monoinfection. AIDS. 2017;31(11):1621-1632.

2. Vodkin I, Valasek MA, Bettencourt R, Cachay E, Loomba R. Clinical, biochemical and histological differences between HIV-associated NAFLD and primary NAFLD: a case-control study. Aliment Pharmacol Ther. 2015;41(4):368-378.

3. Stanley TL, et al. Effects of tesamorelin on non-alcoholic fatty liver disease in HIV: a randomised, double-blind, multicentre trial. Lancet HIV. 2019;6(12):e821-e830.

4. Liberzon A, Birger C, Thorvaldsdóttir H, Ghandi M, Mesirov JP, Tamayo P. The Molecular Signatures Database (MSigDB) hallmark gene set collection. Cell Syst. 2015;1(6):417-425.

5. Subramanian A, et al. Gene set enrichment analysis: a knowledge-based approach for interpreting genome-wide expression profiles. Proc Natl Acad Sci U S A. 2005;102(43):15545-15550.

6. Moon H, Cho K, Shin S, Kim DY, Han KH, Ro SW. High risk of hepatocellular carcinoma development in fibrotic liver: role of 
the Hippo-YAP/TAZ signaling pathway. Int J Mol Sci. 2019;20(3):E581.

7. Hoang SA, et al. Gene expression predicts histological severity and reveals distinct molecular profiles of nonalcoholic fatty liver disease. Sci Rep. 2019;9(1):12541.

8. Patel J, et al. Association of noninvasive quantitative decline in liver fat content on MRI with histologic response in nonalcoholic steatohepatitis. Therap Adv Gastroenterol. 2016;9(5):692-701.

9. Simões ICM, Fontes A, Pinton P, Zischka H, Wieckowski MR. Mitochondria in non-alcoholic fatty liver disease. Int J Biochem Cell Biol. 2018;95:93-99.

10. Teodoro JS, Rolo AP, Duarte FV, Simões AM, Palmeira CM. Differential alterations in mitochondrial function induced by a choline-deficient diet: understanding fatty liver disease progression. Mitochondrion. 2008;8(5-6):367-376.

11. Koliaki C, et al. Adaptation of hepatic mitochondrial function in humans with non-alcoholic fatty liver is lost in steatohepatitis Cell Metab. 2015;21(5):739-746.

12. Ajith TA. Role of mitochondria and mitochondria-targeted agents in non-alcoholic fatty liver disease. Clin Exp Pharmacol Physiol. 2018;45(5):413-421.

13. Short KR, Moller N, Bigelow ML, Coenen-Schimke J, Nair KS. Enhancement of muscle mitochondrial function by growth hormone. J Clin Endocrinol Metab. 2008;93(2):597-604.

14. Pérez R, et al. Mitochondrial protection by low doses of insulin-like growth factor- I in experimental cirrhosis. World J Gastroenterol. 2008;14(17):2731-2739.

15. Makimura H, Murphy CA, Feldpausch MN, Grinspoon SK. The effects of tesamorelin on phosphocreatine recovery in obese subjects with reduced GH. J Clin Endocrinol Metab. 2014;99(1):338-343.

16. Crespo J, et al. Gene expression of tumor necrosis factor alpha and TNF-receptors, p55 and p75, in nonalcoholic steatohepatitis patients. Hepatology. 2001;34(6):1158-1163.

17. Wieckowska A, Papouchado BG, Li Z, Lopez R, Zein NN, Feldstein AE. Increased hepatic and circulating interleukin-6 levels in human nonalcoholic steatohepatitis. Am J Gastroenterol. 2008;103(6):1372-1379.

18. Deepak D, et al. The influence of growth hormone replacement on peripheral inflammatory and cardiovascular risk markers in adults with severe growth hormone deficiency. Growth Horm IGF Res. 2010;20(3):220-225.

19. Makimura $\mathrm{H}$, et al. Metabolic effects of a growth hormone-releasing factor in obese subjects with reduced growth hormone secretion: a randomized controlled trial. J Clin Endocrinol Metab. 2012;97(12):4769-4779.

20. Liu Z, et al. Growth hormone control of hepatic lipid metabolism. Diabetes. 2016;65(12):3598-3609.

21. Li BH, He FP, Yang X, Chen YW, Fan JG. Steatosis induced CCL5 contributes to early-stage liver fibrosis in nonalcoholic fatty liver disease progress. Transl Res. 2017;180:103-117.e4.

22. Hanson A, et al. Chemokine ligand 20 (CCL20) expression increases with NAFLD stage and hepatic stellate cell activation and is regulated by miR-590-5p. Cytokine. 2019;123:154789.

23. Friedman SL, et al. A randomized, placebo-controlled trial of cenicriviroc for treatment of nonalcoholic steatohepatitis with fibrosis. Hepatology. 2018;67(5):1754-1767.

24. Cordero-Espinoza L, Huch M. The balancing act of the liver: tissue regeneration versus fibrosis. J Clin Invest. 2018;128(1):85-96

25. Alkhouri N, Carter-Kent C, Feldstein AE. Apoptosis in nonalcoholic fatty liver disease: diagnostic and therapeutic implications Expert Rev Gastroenterol Hepatol. 2011;5(2):201-212.

26. Fabregat I, et al. TGF- $\beta$ signalling and liver disease. FEBS J. 2016;283(12):2219-2232.

27. Dooley S, ten Dijke P. TGF- $\beta$ in progression of liver disease. Cell Tissue Res. 2012;347(1):245-256.

28. Nishizawa H, et al. IGF-I induces senescence of hepatic stellate cells and limits fibrosis in a p53-dependent manner. Sci Rep. 2016;6:34605.

29. Sobrevals L, et al. Insulin-like growth factor I gene transfer to cirrhotic liver induces fibrolysis and reduces fibrogenesis leading to cirrhosis reversion in rats. Hepatology. 2010;51(3):912-921.

30. Hao CN, et al. Insulin-like growth factor-1 receptor activation prevents hydrogen peroxide-induced oxidative stress, mitochondrial dysfunction and apoptosis. Apoptosis. 2011;16(11):1118-1127.

31. Cast A, et al. Liver proliferation is an essential driver of fibrosis in mouse models of nonalcoholic fatty liver disease. Hepatol Commun. 2019;3(8):1036-1049.

32. Vansaun MN, Mendonsa AM, Lee Gorden D. Hepatocellular proliferation correlates with inflammatory cell and cytokine changes in a murine model of nonalchoholic fatty liver disease. PLoS One. 2013;8(9):e73054.

33. Zhang Y, et al. E2F1 is a novel fibrogenic gene that regulates cholestatic liver fibrosis through the Egr-1/SHP/EID1 network. Hepatology. 2014;60(3):919-930.

34. Denechaud PD, et al. E2F1 mediates sustained lipogenesis and contributes to hepatic steatosis. J Clin Invest. 2016;126(1):137-150

35. Liao X, et al. Distinct diagnostic and prognostic values of minichromosome maintenance gene expression in patients with hepatocellular carcinoma. J Cancer. 2018;9(13):2357-2373.

36. Fourman LT, et al. Clinical predictors of liver fibrosis presence and progression in HIV-associated NAFLD. Clin Infect Dis. In press.

37. Kleiner DE, et al. Design and validation of a histological scoring system for nonalcoholic fatty liver disease. Hepatology. 2005;41(6):1313-1321

38. Kim D, Paggi JM, Park C, Bennett C, Salzberg SL. Graph-based genome alignment and genotyping with HISAT2 and HISAT-genotype. Nat Biotechnol. 2019;37(8):907-915.

39. García-Alcalde F, et al. Qualimap: evaluating next-generation sequencing alignment data. Bioinformatics. 2012;28(20):2678-2679.

40. Liao Y, Smyth GK, Shi W. featureCounts: an efficient general purpose program for assigning sequence reads to genomic features. Bioinformatics. 2014;30(7):923-930.

41. Patano L. DEGreport: report of DEG analysis. R website. http://1pantano.github.io/DEGreport/. Published 2017. Updated 2020

42. Patro R, Duggal G, Love MI, Irizarry RA, Kingsford C. Salmon provides fast and bias-aware quantification of transcript expression. Nat Methods. 2017;14(4):417-419.

43. Love MI, Huber W, Anders S. Moderated estimation of fold change and dispersion for RNA-seq data with DESeq2. Genome Biol. 2014;15(12):550.

44. Soneson C, Love MI, Robinson MD. Differential analyses for RNA-seq: transcript-level estimates improve gene-level inferences 
F1000Res. 2015;4:1521.

45. Kolde R. Package 'pheatmap.' CRAN website. R package. https://cran.r-project.org/web/packages/pheatmap/pheatmap.pdf. Published December 26, 2018. Updated January 4, 2019.

46. Uhlen M, et al. Towards a knowledge-based Human Protein Atlas. Nat Biotechnol. 2010;28(12):1248-1250.

47. Hoshida Y, et al. Gene expression in fixed tissues and outcome in hepatocellular carcinoma. N Engl J Med. 2008;359(19):1995-2004.

48. Zhang DY, et al. A hepatic stellate cell gene expression signature associated with outcomes in hepatitis C cirrhosis and hepatocellular carcinoma after curative resection. Gut. 2016;65(10):1754-1764.

49. Wang Y, et al. Comprehensive molecular characterization of the Hippo signaling pathway in cancer. Cell Rep. 2018;25(5):1304-1317.e5. 